\title{
La práctica pedagógica apoyada en las TIC como estrategia para el fortalecimiento de la calidad educativa ${ }^{1}$
}

\section{The pedagogical practice supported by ICT as a strategy for the strengthening of educational quality}

DOI: http://dx.doi.org/10.17981/cultedusoc.9.3.2018.57

\author{
Artículo de investigación. Fecha de recepción: 15/06/2018. Fecha de aceptación: 27/11/2018 \\ Diana Florez Martínez"2; \\ Juan Florez Díaz; Dennis Ardila Simanca y Sofía Ruiz Vanegas ${ }^{3}$ \\ Institución Educativa Departamental Rural Enrique Quintero Jaimes \\ dianaflorez31@hotmail.com
}

Para citar este artículo:

Florez, D., Florez, J., Ardila, D. y Ruiz, S. (2018). La práctica pedagógica apoyada en las TIC como estrategia para el fortalecimiento de la calidad educativa. Cultura. Educación y Sociedad 9(3), 485-492. DOI: http://dx.doi.org/10.17981/cultedusoc.9.3.2018.57

\section{Resumen}

El presente artículo presenta los resultados derivados de la investigación acerca de la educación como uno de los pilares que permite el progreso de la sociedad, por lo que es fundamental que la calidad de la misma sea alta para garantizar el buen desempeño de los estudiantes en el aula de clases y adecuados niveles de aprendizaje que se evidencian en las distintas pruebas de estado, por lo que se proponen las Tecnologías de la Información y la Comunicación (TIC) como herramientas posibilitadoras de espacios de aprendizaje significativo en el que los estudiantes se vean motivados y dispuestos de manera positiva a ser agentes activos en la construcción de su propio conocimiento, evidenciándose mejores resultados en su proceso de formación. El propósito de la presente investigación es fomentar prácticas pedagógicas mediante ambientes de aprendizaje apoyados en las TIC para el fortalecimiento de la calidad educativa.

Palabras clave: Prácticas pedagógicas, tecnologías de la comunicación e información, calidad de la educación

\section{Abstract}

This article presents the results derived from research about education as one of the pillars that allows the progress of society, so it is essential that the quality of it is high to ensure the good performance of students in the classrooms and appropriate levels of learning that are evident in the different state tests, so that Information and Communication Technologies (ICT) are proposed as enabling tools for significant learning spaces in which students are motivated and willing in a positive way to be active agents in the construction of their own knowledge, evidencing better results in their training process. The purpose of this research is to promote pedagogical practices through learning environments supported by ICT for the strengthening of educational quality.

Keywords: Pedagogical practices, communication and information technologies, quality of education

1 Este artículo ha sido derivado del Proyecto Fortalecimiento de la Cultura Ciudadana y Democrática en CT+I a través de la IEP apoyada en TIC en el Dpto. del Magdalena.

2 Líder del grupo de investigación

3 Docentes de la Institución Educativa Departamental Rural Enrique Quintero Jaimes.

- The author; licensee Universidad de la Costa - CUC. 


\section{Introducción}

La educación es uno de los pilares que permite el progreso de la sociedad, por lo que es fundamental que el esfuerzo sea de alta calidad para garantizar el buen desempeño de los estudiantes en el aula de clases y adecuados niveles de aprendizaje, lo cual debe hacerse evidente en las distintas pruebas que aplica el estado. La calidad educativa es responsabilidad de todos los miembros del plantel, quienes de manera comprometida y responsable deben apropiarse de los procesos de enseñanza-aprendizaje.

En la Institución Educativa Departamental (IED), Rural Enrique Quintero Jaimes se evidencia un bajo rendimiento en las Pruebas Saber que buscan medir la calidad educativa, lo cual, de acuerdo a los docentes del plantel responde a una falta de motivación hacia los procesos académicos por parte de los estudiantes, lo cual obstaculiza el desarrollo eficaz de los mismos e impide que se alcance los logros fijados en el currículo, todo la cual se evidencia porque fingen estar enfermos para no entrar a clases, mantienen relaciones distantes con los profesores, generan brotes constantes de indisciplina, no cumplen con sus responsabilidades académicas, exteriorizan gestos de rechazo hacia algunos docentes y algunas actividades.

En este sentido, se proponen las Tecnologías de la Información y la Comunicación (TIC), como herramientas posibilitadoras de espacios de aprendizaje significativo en el que los estudiantes se vean motivados y dispuestos de manera positiva a ser agentes activos en la construcción de su propio conocimiento, evidenciándose mejores resultados en su proceso de formación. Al respecto, Marín, Inciarte, Hernández y Pitre (2017), señalan la pertinencia de la mediación de las TIC, por cuanto permiten fortalecer los procesos pedagógicos a través de estrategias de integración e innovación.

La educación se contempla como un derecho fundamental de todas las personas que en la Declaración Universal de los Derechos Humanos (ONU, 2015), en las orientaciones centrales de la UNESCO (2017), y también en el enunciado de los Objetivos de Desarrollo Sostenible para el 2030. Según esta última programación, se busca garantizar para todas las personas del mundo el acceso a una educación inclusiva y equitativa de calidad, y se reconoce la relevancia que posee en el marco de los gobiernos nacionales e internacionales, la educación es base fundamental y es vista como una poderosa herramienta de transformación económica y social.

A nivel nacional, a partir de lo propuesto en el Plan Nacional Decenal de Educación 2016-2026 (MEN, 2017), se busca desarrollar un sistema educativo de alta calidad que fomente el desarrollo en todos los niveles del país, y una sociedad construida con valores como el respeto, la justicia, la equidad y la tolerancia frente a las diferencias. Desde esta propuesta, "Una educación de calidad es aquella que propone y alcanza fines pertinentes para las personas y las comunidades en el contexto de una sociedad en continuo progreso y que la hace competitiva en el contexto mundial" (MEN, 2017, p. 15).

De acuerdo a Ausebel (citado por Rodríguez, 2011), se puede distinguir entre dos tipos de aprendizaje: el repetitivo y el significativo. El primero es un aprendizaje superficial, en el que no se logra establecer una conexión entre los nuevos conocimientos y los ya previamente adquiridos, mientras que el aprendizaje significativo se da de manera profunda, el conocimiento adquirido es duradero y fácilmente asequible. Este autor plantea que para que este tipo de aprendizaje 
ocurra, es necesario que el contenido enseñado sea potencialmente significativo, que tenga continuidad con las temáticas trabajadas anteriormente y que el estudiante tenga una disposición positiva hacia el proceso de enseñanza-aprendizaje, y estas tres condiciones propician un aprendizaje significativo y, por ende, una educación de calidad.

Una de las maneras en que se busca medir en forma periódica cómo se encuentran los niveles educativos en el país, y si este ha sido significativo o no, son las Pruebas Saber realizadas a nivel nacional, en los cursos $3^{\circ}, 5^{\circ}$ y $9^{\circ}$, con el objetivo principal de,

"determinar en qué grado los estudiantes de educación básica se acercan al logro de los resultados esperados, según los estándares básicos de competencias definidos por el Ministerio de Educación Nacional. Los resultados de esta evaluación permiten que las instituciones educativas, las secretarías y el Ministerio de Educación Nacional (MEN) construyan planes de mejoramiento en sus respectivos ámbitos de actuación y valoren los avances en el tiempo" (ICFES, 2012, p. 4).

En este sentido, cuando en una institución educativa se evidencia a través de estas pruebas o cualquier otro medidor del nivel de educación, que estos se encuentran bajos o deficientes en relación al grado de los estudiantes, es responsabilidad de la misma trabajar de la mano con todos sus miembros para establecer estrategias de intervención, con el fin de cumplir con la agenda nacional e internacional relacionada con la calidad de la educación.

En relación a lo anterior, Herrera (Citado por Molina, 2013) plantea que,

"uno de los grandes retos en la actualidad es mejorar la calidad de la educación. Procurar que los estudiantes aprendan más y de mejor forma es la preocupación de docentes e instituciones educativas. Para ello es necesario que los alumnos cuenten con ambientes de aprendizaje más efectivos y didácticos; entornos educativos que les permitan desarrollar sus habilidades para pensar y su capacidad para aprender. En este sentido, los ambientes virtuales de aprendizaje representan nuevas posibilidades de acceso a la educación" (p. 242).

Así mismo, la UNESCO (2013), plantea que al comenzar el siglo XXI, el acceso a una educación de calidad, se enfrenta a un contexto de cambio paradigmático, como derecho fundamental de todas las personas. El desarrollo que han alcanzado las Tecnologías de la Información y la Comunicación (TIC), en los últimos años, le plantea desafíos al sistema educacional, se requiere actualización de prácticas y contenidos para que sean pertinentes a la nueva sociedad de la información. Esta actualización implica una flexibilidad y transformación pedagógica, para incorporar las TIC al aula y al currículum escolar.

Desde el MEN (2017) también se proponen las Tecnologías de la Información y la Comunicación (TIC) como herramientas para la contribución al acceso universal a la educación, la igualdad en la instrucción de contenidos, el ejercicio en los procesos de enseñanza - aprendizaje con garantías de calidad y el desarrollo de docentes más capacitados.

Las TIC, de acuerdo a Ramírez (2010), son herramientas que posibilitan dinamizar las prácticas pedagógicas a través de procesos y actividades innovadores que permiten la adquisición de aprendizajes significativos por parte de los estudiantes, dinamizan las prácticas pedagógicas gracias a sus características, incentivan la innovación y la creatividad de estudiantes y docentes. Este proceso requiere que los estudiantes desarrollen procesos de adaptación y socialización para la interacción en el medio educativo. En 
este sentido, Vallejo (2011) enfatiza la necesidad de consolidar mecanismos de adaptación desde el plano personal como rasgo distintivo, centrado en valores, y participando propositivamente en el cumplimiento de las tareas que se correspondan con su formación integral. El logro de este propósito puede ser viabilizado desde la integración de las TIC al proceso didáctico.

La implementación de las TIC en el contexto educativo favorece el desarrollo de competencias en tanto motiva a los estudiantes, en función de la dinámica global actual (Avendaño y Martínez, 2013). En este sentido, el apoyo de los docentes y papel activo de los docentes y las prácticas pedagógicas mediadas por el uso de las TIC contribuye a la disposición positiva de los estudiantes, en tanto estas herramientas los han marcado su interacción con el mundo desde edades muy tempranas, por lo que son considerados nativos digitales (Prensky, 2001).

El uso de las TIC en los procesos de enseñanza-aprendizaje brinda a los estudiantes un papel activo y mayor autonomía en la adquisición de su conocimiento, lo que tiene como resultado un aprendizaje con sentido y no repetitivo (Cortes Orozco, Luna y Martínez, 2015). La utilización de las TIC fomenta el pensamiento crítico, reflexivo, eficaz e innovador en los estudiantes (Kutscher y St. Pierre, 2001). A decir de Hernández, Alvarado y Velazco (2013) las TIC representan un espacio propicio para fortalecer los procesos de participación; a través de la mediación de las redes que permiten la interacción e intercambio de opiniones.

Sin embargo, se ha evidenciado que las TIC han sido poco efectivas por su inadecuada inclusión en las prácticas pedagógicas. Tal como lo plantea Area-Moreira (2008), pese al esfuerzo de las instituciones por tener acceso a los recursos tecnológicos, estos no han sido adaptados a los currículos de enseñanza tradicional, por lo que no se evidencia un impacto positivo en los procesos de aprendizaje.

De acuerdo a Fernández, Rodríguez y Vidal (2004) y Sigalés (citado por Coll, 2008), lo anterior responde a que los docentes siguen utilizando las mismas estrategias tradicionales solo que basadas en las TIC, por ejemplo la implementación de diapositivas para presentar los contenidos, pero se requiere una visión más activa, en la que los docentes las utilicen como herramientas de exploración, que fomenten el trabajo autónomo y colaborativo de sus estudiantes, por lo que desde el presente trabajo se busca fomentar su inclusión a las prácticas pedagógicas de manera adecuada, para así contribuir al mejoramiento de los niveles de calidad a nivel institucional.

\section{Metodología}

Este trabajo es un producto derivado del estudio sobre la práctica pedagógica apoyada en las TIC como estrategia para el fortalecimiento de la calidad educativa. Desarrollado en la Institución Educativa Departamental Rural Enrique Quintero Jaimes.; su objetivo principal ha sido dinamizar los procesos de enseñanzaaprendizaje, en tanto aumenta la motivación de los estudiantes en la construcción de conocimiento, lo que da cuenta de la mejoría de los procesos y de la calidad educativa.

Este estudio contempla en su metodología una metodología cualitativa, teniendo en cuenta que esta "se enfoca a comprender y profundizar los fenómenos, explorándolos desde la perspectiva de los participantes en un ambiente natural y en relación con el contexto" (Hernández, Collado y Baptista, 2010, p. 364).

La unidad de análisis estuvo conformada por los estudiantes de la Institución Educativa Departamental Rural Enrique 
Quintero Jaimes. En función del análisis de los resultados institucionales de las Pruebas Saber se realizó, en primer lugar, la observación de las prácticas pedagógicas que estaban implementando los docentes para la enseñanza de los distintos contenidos, con el fin de observar cómo las estaban llevando a cabo y la actitud y disposición de los estudiantes frente a las mismas.

Sobre los instrumentos y procedimientos, se aplicaron encuestas y se realizaron una serie de entrevistas semiestructuradas con los estudiantes, para conocer su percepción frente a dichas prácticas, trabajadas por sus docentes en las clases, teniendo en cuenta el uso de estrategias innovadoras, el uso de ejemplos para explicar, la implementación de herramientas tecnológicas a las clases y cómo se sienten frente a estas.

Finalmente se realizaron talleres y mesas de trabajo con estudiantes y docentes para el diseño de nuevas estrategias, de manera innovadora y colaborativa, basadas en las TIC, para fortalecer la disposición de los estudiantes hacia el aprendizaje y la investigación, mejorar la calidad educativa y el rendimiento institucional en las pruebas saber.

\section{Resultados}

Mediante la observación de las prácticas pedagógicas de los docentes en las aulas de clase se pudo evidenciar que estas no logran los niveles de motivación, lúdica y dinamismo suficiente, para que los estudiantes se sientan comprometidos con el aprendizaje de contenidos, sino que se observan dispersos, por lo que la adquisición de los aprendizajes no se da de manera significativa, lo cual explica el bajo rendimiento de los estudiantes en las pruebas saber.

El desarrollo de talleres con los docentes permitió concientizarlos de la impor- tancia de la implementación de nuevas estrategias para poder mejorar los niveles de aprendizaje en el aula y, por ende, de la calidad de la educación. Así mismo, en las mesas de trabajo tanto estos, como los estudiantes, participaron de manera activa al proponer distintas maneras en que las TIC, dinamizan la experiencia en el desarrollo de contenidos. En este sentido, autores como Betancurt et al (2014), resaltan la necesidad de fortalecer el proceso educativo desde la mediación de las TIC, específicamente a través de sus aportes como herramientas o entornos virtuales de aprendizaje.

Así, mediante el trabajo conjunto entre estudiantes y docentes, se propusieron varias estrategias, tales como la consulta de portales web especializados, el uso de material en formato digital, realizar trabajos en plataformas virtuales, el uso de foros virtuales, tutorías en línea, el desarrollo de blogs y el uso de presentaciones multimedia para exponer trabajos, todo con el fin de contribuir a la motivación de los estudiantes para la construcción de conocimiento significativo.

\section{Conclusiones}

En la era actual de la tecnología resulta indispensable repensar las prácticas pedagógicas tradicionales y el impacto real que están teniendo en la nueva generación, en los nativos digitales, que a través de las TIC tienen acceso a una gran cantidad de contenido y en múltiples formatos, por lo que las estrategias utilizadas en las aulas ya no les generan motivación suficiente. Teniendo en cuenta lo anterior, es pertinente plantear según Karam, Buitrago, Fagua y Romero (2013), que las Tecnologías de la Información y la Comunicación (TIC) son un actor fundamental y parte de la cultura académica.

La inclusión de las TIC al contexto educativo permite un acercamiento real 
con los estudiantes, quienes se sienten más incluidos, comprometidos y motivados a trabajar con estas herramientas los contenidos desarrollados en las clases, en tanto crean un ambiente mucho más dinámico, eficaz y óptimo para el aprendizaje. Además, les da mayor autonomía en sus procesos de aprendizaje y en la construcción de su propio conocimiento, adquiriendo así las clases un modelo pedagógico más desarrollista, siendo que también permite que exploren y desarrollen otro tipo de capacidades que con la metodología de enseñanza tradicional no era posible trabajar.

Por último y teniendo en cuenta la inclusión de la tecnología también es importante permitir a los estudiantes tomar el control de su proceso dentro de la institución no solo permite que aprendan mejor y más rápido, de manera significativa, sino que también genera un ambiente propositivo, participativo, didáctico y de mejores relaciones entre los estudiantes y sus docentes, favoreciendo significativamente el desarrollo de las prácticas de convivencia escolar (De la Concepción, 2015).

Finalmente, y de acuerdo con los resultados obtenidos en este estudio, se refuerza lo planteado por Ricardo, et al. (2013), como un desafío para la educación en Colombia, se perfila una ruta de apropiación de las TIC en el desarrollo profesoral docente, una apropiación personal y una profesional, con la intención de utilizarlas pedagógicamente en diversos contextos educativos. De hecho la orientación emanada del MEN (2008), sobre el manejo básico de herramientas de información y comunicación en favor el desarrollo y fortalecimiento de otras competencias básicas para el desarrollo humano, sigue esperando por su cumplimiento. Son los docentes los que tienen el desafío de abrir nuevos horizontes a la tarea educativa, dinamizándola y haciéndola más pertinente a los momentos actuales.

\section{Referencia}

Area-Moreira, M. (2008). La innovación pedagógica con TIC y el desarrollo de las competencias informacionales $\mathrm{y}$ digitales. Revista de Investigación en la Escuela, (64), 5-17. Recuperado de http://www.investigacionenlaescuela. es/articulos/64/R64_1.pdf

Avendaño, I. y Martínez, D. (2013). Competencia lectora y el uso de las nuevas tecnologías de la información y comunicación. Revista Escenarios, 11(1), 7-22.

Betancur, S., Carmona, L., Contreras, R., Karam, J., Maestre, N., Romero, Y. y Uribe, S. (2014). Videojuegos y tic como Estrategias Pedagógicas: Formación para el uso seguro de internet. Cultura Educación y Sociedad, 5(1). 91-107. Recuperado de https://revistascientificas.cuc.edu.co/culturaeducacionysociedad/article/view/997

Coll, C. (2008). Aprender y enseñar con las TIC: expectativas, realidad y potencialidades. Boletín de la Institución Libre de Enseñanza, 72,(1). 17-40

Cortés, J., Orozco, G., Luna, V. y Martínez, A.. (2015). El Aprendizaje Distribuido, primer paso a la virtualidad en la Enseñanza en el Nivel Universitario. Revista Iberoamericana de Producción Académica y Gestión Educativa, 2(3). 1-14. Recuperado de http://www.pag.org.mx/index.php/ PAG/article/view/263/309

De la Concepción, A. (2015). Concepciones sobre participación de niñas, niños y adolescentes: Su importancia en la construcción de la convivencia escolar. Cultura Educación y Sociedad, 6(2), 9-28. Recuperado de https://revistascientificas.cuc.edu.co/culturaed u c a cionys ocied a d / a r ticle/ download/840/pdf_105/ 
Fernández, M., Rodríguez, J. y Vidal, M. (2004). La influencia de las TIC en el desarrollo organizativo y profesional de un centro de Primaria. Barcelona: EDUTEC.

Hernández, J., Alvarado, L. y Velazco, J. (2013). Participación ciudadana, tecnologías de información-comunicación y opinión. Revista Científica Teorías, Enfoques y Aplicaciones en las Ciencias Sociales, 5 (11) 25-34. Recuperado de https://dialnet.unirioja.es/servlet/ articulo?codigo $=4736149$

ICFES. (2012). Guía para la lectura e interpretación de los reportes de resultados institucionales de la aplicación muestral de 2011. Bogotá D.C.: Ministerio de Educación Nacional.

Karam, J., Buitrago, A., Fagua, A. y Romero, Y. (2013). Estrategias de gamificación aplicadas al diagnóstico de la incorporación pedagógica de las TIC en una comunidad académica. Cultura, Educación y Sociedad, 4(1), 55-74.

Kustcher, N., y St Pierre, A. (2001). Pedagogía e internet. Aprovechamiento de las nuevas tecnologías. México, D.F.: Editorial Trillas.

Marín, F., Inciarte, A., Hernández, H y Pitre, R. (2017). Estrategias de las Instituciones de Educación Superior para la Integración de las Tecnología de la Información y la Comunicación y de la Innovación en los Procesos de Enseñanza. Un Estudio en el Distrito de Barranquilla, Colombia. Formación universitaria, 10(6), 29-38. https:// dx.doi.org/10.4067/S0718 50062017000600004

Ministerio de Educación Nacional-MEN. (2008). Apropiación de TIC en el desarrollo profesional docente v2.0. Programa nacional de uso de medios y nuevas tecnologías. Bogotá: Ruta de desarrollo profesional docente para el uso de nuevas tecnologías. Bogotá, D.C.: MEN.
Ministerio de Educación Nacional-MEN. (2017). Plan Nacional decenal de Educación 2016-2026. Bogotá, D.C.: MEN. Recuperado de http://www.plandecenal.edu.co/cms/media/herramientas/ PNDE\%20FINAL_ISBN\%20web.pdf

Molina, G., Tarín, A. y Sevila, V. (2013). Diseño de la formación de postgrado E-Learning: el caso del Máster en Liderazgo Político y Dirección de Organizaciones Públicas. En, M. Tortosa XI Jornadas de Redes de Investigación en Docencia Universitaria. Retos de futuro en la enseñanza superior: Docencia e investigación para alcanzar la excelencia académica (2420-2431). Universidad de Alicante, Alicante, España

ONU. (2015). Declaración Universal de los Derechos Humanos. Recuperado de http://www.un.org/es/documents/udhr/ UDHR_booklet_SP_web.pdf

Prensky, M. (2001). Digital Natives, Digital Immigrants. On the Horizon, (9). 1-6

Ramírez, C. (2010). Las TICS en el Aula. Granada: NNTT.

Ricardo, C., Borjas, M., Velásquez, I., Colmenares, J. y Serje, A. (2013). Caracterización de la integración de las TIC en los currículos escolares de instituciones educativas en Barranquilla. $R e$ vista Zona próxima. 18(en línea).

Rodríguez, M. (2011). La teoría del aprendizaje significativo: una revisión aplicable a la escuela actual. Revista Electrónica d'Investigació i Innovació Educativa $i$ Socioeducativa, 3(1). 29. 50

UNESCO. (2013). Enfoques estratégicos sobre las TICs en Educación en América Latina y el Caribe. Santiago: Unesco. Recuperado de http://www. unesco.org/new/fileadmin/MULTIMEDIA/FIELD/Santiago/images/ticsesp.pdf 
UNESCO. (2017). La educación transforma vidas.París: Unesco. Recuperado de http://unesdoc.unesco.org/images/ 0024/002472/247234s.pdf
Vallejo, O. (2016). Adaptación laboral: Factor clave para el rendimiento y la satisfacción en el trabajo. Cultura Educación y Sociedad 2(1), 171 - 176. Recuperado de https://revistascientificas.cuc.edu.co/ culturaeducacionysociedad/article/ view/946 